\title{
MACROEVOLUTIONARY PATTERNS OF BODY PLAN CANALIZATION IN EUARTHROPODS
}

Cédric Aria

\section{SUPPLEMENTARY INFORMATION}

\section{Supplementary Methods}

Datasets. The source dataset (FID, see Dataset S2), improved from previous versions (Aria and Caron 2017a, Aria and Caron 2017b, Vannier et al. 2018), was initially designed to be used optimally for multivariate analyses. This required, notably, a reduction as much as possible of the missing data, which can severely skew morphospace coordinates (see, e.g., Lloyd 2016; see also outlier cases in Aria and Caron 2015), despite the use of algorithms minimizing this effect (e.g. here, daisy, from the R package cluster). This bias was further reduced by avoiding to use disparity metrics based on variance (as in Briggs et al. 1992) and by systematically resampling the data so as to simulate larger distributions. For this reason, it would be detrimental rather than beneficial for the analysis to have a broader sampling encompassing characters that can be coded only in extant taxa, and which would therefore generate massive proportions of missing entries (e.g., ca. 70\% in Legg et al. 2013 including inapplicable states). This missing information was compensated here by implementing topological constraints based on the results of phylogenetic analyses making use of these extant-only data, and further reinforced by constraints based on molecular results.

It follows that the reconstruction of disparity is also based on this subset of characters, most of which applicable to fossils. However, the very concept of disparity, as opposed to diversity, relies on the view that morphology is arranged into (developmental) levels, with higher divisions corresponding to more fundamental differentiations. There is therefore a rationale supporting the evolutionary significance of the subset of architectural characters defining the main variations in the euarthropod body plan. In order to evaluate this hypothesis, the results obtained with this work's dataset were compared with those of the independently-coded dataset of Lee et al. (2013) (and largely based on Rota-Stabelli et al. 2011), which includes a comprehensive set of morphological characters for extant taxa (EOD).

It is also difficult to determine the boundaries for an ideally representative pool of fossil taxa, but the same principle of disparity mentioned above also applies in this case: adding more trilobite taxa, for instance, is unlikely to either refine or complement a morphospace plotted at the level of Euarthropoda. Resampling helps simulating the morphological variation absent from the source data. Like any form of modelling, it is limited by the approximation of its estimated distribution, here either a jackknifing of the original data (ranges metrics) or Gaussian (hypervolumes).

The general methodology used here is similar to Aria and Caron (2015) and Caron and Aria (2017), although there was no culling of entirely separate clusters and outliers from the morphospace. Non euarthropod taxa (except for Isoxyidae) and characters thereby becoming constant were excluded from the phylogenetic dataset (characters becoming autapomorphic were kept; see Dataset S2). See below for details.

The fossil-inclusive dataset also initially contained a relatively large number of polymorphic entries (e.g. "(01)"). However, algorithms computing dissimilarity matrices are not designed to handle polymorphic entries in morphological datasets. To circumvent this limitation, 
taxa with polymorphic entries were duplicated to express the alternative coding separately (see Dataset S2). The eigenvalues were then averaged on each axis between the duplicates to obtain a single point for each taxon. In case of polymorphic entries in $n$ different characters, taxa were not duplicated $\mathrm{n}$ times for practicality, and only one duplicate was used to represent the alternative states. Occurrences of third polymorphic state, very rare, were not accounted for.

The coding of wings. FID was initially coded and tested without a presence/absence of wings character, because this was autapomorphic to only four taxa and, despite its evolutionary importance, was unlikely to become a determinant character in discriminating euarthropod body plans in their entirety. Indeed, further adding a presence/absence of wings did not alter the results (Supp. Fig. 4B). The separation of hexapods into two distinct clusters in EOD is not clearly driven by any subdivision of Hexapoda either (Supp. Fig. 4A), even if some wing characters could come into play (the inapplicable entries, coded as "?," could then explain the aberrant groupings). In part, this is a limitation of the perspective provided by this methodology, but there are also systematic and biological reasons for this result (see main text).

Impact of topological accuracy on the interpretations. Despite remarkable progress in recent years, resolving the phylogeny of arthropods has always been a challenge, and changes are still to be expected, especially with respect to the placement of fossil groups. It may therefore be posited that the analyses undertaken here are only valid to the extent that the phylogenetic topology they are based on is sound. Although this is in fact true of any macroevolutionary study, necessarily dependent on the available evidence at a given time, there are two lines of arguments that support the robustness of our findings. First, the topology of fossil taxa overall is arguably more robust than a few years back (Legg et al. 2013), owing notably to the revision of hymenocarines and its consequence for the origin of Mandibulata (Aria and Caron 2017a, Vannier et al. 2018) and the significance of other fossils, as well as the use of Bayesian methods. There is thus a degree of confidence in the placement of fossil stem groups that justifies the evolutionary hierarchization of fossil morphogroups, in spite of persisting uncertainties. Second, this hierarchization only has a limited influence on this study's conclusion, insofar as it concerns only those metrics dependent on group order and, within those, only the specific cases of successive fossil morphogroups. To an extent, the evolutionary implications for the disparity of artiopodans, merostomes, megacheirans and hymenocarines would remain the same regardless of phylogenetic placement, as long as they are basal to their extant relatives.

Multivariate analyses. Principal coordinates analysis. Both datasets were converted into dissimilarity matrices via the function dist from the $\mathrm{R}$ package cluster, using a Gower metric and all variables as factors. Principal coordinate analyses were generated via the base function cmdscale.

Dimensionality. Significant dimensionality was investigated via a broken stick model (bstick function in the package vegan).

Determination of morphogroups. Morphogroups were determined a priori based on phylogenetic topology (Fig. 1); most morphogroups were determined by monophyly, but fossil lineages with identifiable body plans and recovered as paraphyletic were also treated as morphogroups. A complementary analysis of the clustering signal intrinsic to the morphospace was performed through a k-means clustering method (cascadeKM function in package vegan), looking for the optimal number of groups between 2 and 10. 
Sum and variance of ranges. Range metrics were calculated via custom functions subtracting the minimum values of the (4) significant axes to their corresponding maximum values, and then summing the results or extracting their variance. This operation was then jackknifed for each morphogroup. Jackknifing was initially preferred to bootstrapping as a less strict resampling method, but subsequent tests showed that results were almost identical between the two approaches.

Hypervolumes. Hypervolume calculations were performed via the $\mathrm{R}$ package hypervolume. These hypervolumes were generated by estimating the Gaussian kernel density of the data over the significant axes. Hypervolume geometry was then determined by the convex hull of these simulated data, from which inter-centroid (of hypervolumes) distances and hypervolume overlaps (Sørensen index) were derived. The latter metrics are used here as additional proxies for the evolution of disparity. Because they constitute a measure of morphological distance between two radiative clusters, they are presented as metrics of "translation" from one body plan to another, this translation corresponding to a gradient of morphological change from one stable adaptive zone to another. Given that it is, in fact, the reverse hypervolume overlap that should be equivalent to a given inter-centroid distance (greater distance=less overlap), this is therefore what is used in this study for simplification. In order to set a reference for comparisons of evolutionary transitions, "phylogenetic transition" points corresponding to the sequence of relevant phylogenetic nodes were assigned to translation values. Thus, for instance, the transitions from megacheirans to artiopodans and from hymenocarines to oligostracans were both assigned to phylogenetic transition \#2, because they both occur between the first successive morphogroups in their respective lineages.

Principal component analysis. A principal component analysis was used on the numeric dataset summarizing disparity metrics and evolutionary rates (Dataset S3; see Fig. 5A). This was performed by applying the prcomp algorithm ( $\mathrm{R}$ base) on a dataset in which non-applicable entries were replaced mean vector values based on the variance of a given variable.

Evolutionary rates and corresponding metrics. Morphological and molecular rates were taken from Lee et al. (2013). Depending on the type of analysis, they were separated between amongmorphogroup rates (or 'transition' rates, i.e., describing branches separating the considered morphogroups), and within-morphogroup rates (or 'internal' rates, i.e., rates present on branches in a given morphogroups). To remain consistent with the structure of the data and the morphospace, rates of terminal branches were excluded from the calculation of withinmorphogroup rates. Including these rates would amount to taking into account all the autapomorphic changes characterizing these terminals, which is incompatible with the structure of both the dataset (most autapomorphic characters are not coded) and the morphospace (which is built around characters that explain the most variance between all taxa and minimizes the impact of more locally significant variations). Transition rates, on the other hand, can provide a different information whether they are taken directly from the branch leading to the considered morphogroups (autapomorphic transition rate, ATR), or indirectly as the average of all branches separating a morphogroup from another (corrected transition rate, CTR). The latter approach considers that morphospace distance is proportional to the evolutionary rates separating morphogroups from their common ancestor. However, an average can severely over- or underestimate evolutionary events resulting from a mixture of very heterogeneous rates. This is for instance the case of hexapods, whose autapomorphic branch is characterized by a very high morphological rate, contrasting starkly with branches connecting them to their last two common 
ancestors (here, Labiocarida and Altocrustacea). For this reason, ATR is a necessary complement to CTR in the comparison of disparity metrics with rates (as illustrated in Fig. 4E). Correlation curves (Fig. 4C, D) are the results of fitting of metrics with rates using various non-linear and linear models, and calculating their corresponding Pearson correlation value. Best fitting models are detailed in captions. Certain macroevolutionary variables were approximated in fossil morphogroups (see Summary Table in Dataset S3) based on these models.

Hierarchical clustering. The UPGMA clustering was chosen as the most representative solution out of the six metrics used ("Single linkage", "Complete linkage", "UPGMA", "UPGMC", "WPGMA", "WPGMC"; see Supp. Fig. 3), and preferred to the equivalent WPGMA because it uses fewer parameters. The clusters were generated using the algorithms dist (based on Euclidean distances) and hclust from the R package vegan.

Character list. The following list is taken from Aria and Caron (2019) and includes the changes made for the present study. Character headings refer to the original publication by $[\mathrm{ACX}]$, where $\mathrm{X}$ is the corresponding number for that character in Aria and Caron (2017a). Descriptions are not carried over here if no change was made. Characters either new since Aria and Caron (2017a), or which have gone through substantial change in overall coding or definition are marked with an asterisk. Some other small corrections were applied to the original matrix but are not reported here in detail.

\section{GENERAL CHARACTERS}

[1] Limbs [AC1]

0 . Absent

1. Present

[2] External cuticular segmentation [AC2]

0 . Absent

1. Present

[3] Type of body segmentation [AC3]

0 . Sclerotized

1. Arthrodized (=tergal)

[4] Calcified cuticle [AC4]

0 . Absent

1. Present

[5] Visual surface with calcified lenses, bounded by circumocular suture

0 . Absent

1. Present

Remark: See Mayers et al. (2018, ch. 12).

[6] Holometaboly [AC6]

0 . Absent 
1. Present

\section{LOBOPODIAN CHARACTERS}

[7] External anteriorization restricted to a single pair of frontalmost appendages [AC7]

0 . Absent

1. Present

[8] Lobopodous limbs [AC8]

0. Absent

1. Present

[9] Type of main lobopodous trunk limb [AC9]

0 . Short, conical, subequal or shorter than trunk width

1. Elongated, slender, longer than trunk width

[10] Flap-like lateral limbs [AC10]

0 . Absent

1. Present

[11] Nodes/tubercles/dermal papillae [AC11]

0 . Absent

1. Present

[12] Differentiation at limb insertion [AC12]

0 . Absent

1. Present

[13] Dorso-lateral sclerites above limb insertion [AC13]

0 . Absent

1. Present

[14] Median spine above limb insertions [AC14]

0 . Absent

1. Present

[15] Lobopod tip (main trunk limb) [AC15]

0 . Double claw

1. Juxtaposed series of claws

2. Pad

[16] Posterior-most single claws [AC16]

0 . Absent

1. Present

[17] Posterior claws pointing anteriad [AC17]

0 . Absent 
1. Present

VISUAL ORGANS

[18] Ocelli as primary ocular units [AC18]

0 . Absent

1. Present

[19] Median eyes [AC19]

0 . Absent

1. Present

[20] Number of median eyes [AC20]

0. 2

1. 3

2. 4

[21] Rhabdomeric lateral eye [AC21]

0 . Very reduced or absent

1. Present and well developed

[22] Type of lateral eyes [AC22]*

0 . Few and simple lenses with cup-shaped retina

1. Faceted (compound)

2. Stemmata

[23] Type of corneagenous cells [AC23]

0 . Many

1. Two

[24] Tetraconate condition [AC24]

0 . Absent

1. Present

[25] Number of nested optic neuropils [AC25]

0.1

1. 2

2. 3

[26] Multi-layered rhabdomeres [AC26]

0. Absent

1. Present

[27] Star-shaped rhabdomeres [AC26]

0. Absent

1. Present 
[28] Lateral compound eye topology [AC27]*

0 . Projecting laterally beneath the head shield or through a notch

1. Projecting dorsally and embedded within the shield

2. Projecting latero-ventrally close to the medial axis, possibly accommodated with bulges in shield

Remark: See Mayers et al. (2018, ch. 11).

[29] Opthalmic ridges [AC28]

0 . Absent

1. Present

[30] Lateral eyes pedunculate [AC29]

0 . Absent

1. Present

[31] Peduncular lobes [AC67]

0. Absent

1. Present

Remark: See Vannier et al. (2018) for a discussion of that character.

[32] Pedunculate eyes large and ovate, part of a prominent ocular segment projecting anteriad [AC30]

0 . Absent

1. Present

HEAD AND CEPHALIC CHARACTERS

[33] Somital head (as tagma I) defined by series of appendages and/or external segmentation [AC31]

0 . Absent (only anteriormost defines head)

1. Present

[34] Somites defining anteriormost tagma* [AC31]

0.4

1. 5

2. 6

3. 7

4. 8

Remark: Habeliidans and relevant synziphosurines are coded for octasomitic heads following Aria and Caron (2017b).

[35] Tergite of the ocular (protocerebral) somite [AC127]*

0 . Absent

1. Present 
Remark: We consider here that the chelicerate epistome is homologous to other types of frontal sclerites among euarthropods.

[36] Tergite of the ocular (protocerebral) somite, type [AC128]

0 . Rounded

1. Sub-triangular

[37] Supernumerary frontal sclerite attached to the ocular tergite [AC128]

0 . Absent

1. Present

Remark: This character codes for the so-called epipharyngeal sclerite attached to the epistome in some chelicerates.

[38] Tergal sclerotization of the post-ocular somite [AC33]

0 . Absent

1. Present

[39] Tergal sclerotization type [AC34]

0 . Tergites with posterior expansion over at least some trunk segments (carapace)

1. Tergites with limited expansion, cephalic tergites all fused and articulating or connecting with first trunk segment (shield)

[40] Carapace type [AC35]

0 . Bivalved

1. Plate

[41] Bivalved carapace type*

0. Type I: Sub-straight cross-section, covering body dorsally

1. Type II: Convex cross-section, enveloping body laterally

[42] Shape of carapacal valves*

0. Symmetrical respective to sagittal axis, ventral margins tight ('Isoxys type')

1. Asymmetrical respective to sagittal axis, antero-ventral margin tight, postero-ventral margin ample ('Canadaspis type')

2. Symmetrical respective to sagittal axis, ventral margins ample ('Branchiocaris type')

3. Variable (ostracods)

[43] Ventral closure of carapace*

0 . Absent

1. Present

Remark: 'Odaraiids' (Odaraia and Nereocaris) are characterized by carapacal valves that fully enclose the body, with ventral margins extensively covering the underside of the body. We recognize a similar condition in ostracods. 
[44] Type II bivalved configuration [AC36]*

0 . Unfused along most of dorsal margin

1. Fused along most of dorsal margin

Remark: Among hymenocarines, we consider that Odaraia and Waptia present an extensive fusion of the valves with effacement of the median fold.

[45] Covering of the type II bivalved carapace (when body fully extended antero-posteriorly) [AC37]

0 . At least two thirds of body length

1. Cephalothorax

[46] Frontal and lateral extensions of cephalic shield*

0 . Limited; tergites not raised or expanding laterally

1. Distinct and well-developed; shield forms anterior and/or lateral extensions

[47] Articulation of posterior margin of shield with first trunk segment [AC39]

0 . Tergal overlap

1. Occipital closure

[48] Segmental impression in shield [AC40]

0 . Absent

1. Present

[49] Occipital lobe [AC41]

0 . Absent

1. Present

[50] Pair of occipital carinae [AC42]

0 . Absent

1. Present

[51] Anterior reduction of segments and/or appendages [AC43]

0 . Absent

1. Present

[52] Compaction of the cephalic unit [AC44]

0 . Absent

1. Present

[53] Cephalic doublure [AC45]

0 . Absent

1. Present

[54] Cephalic kinesis [AC46] 
0. Absent

1. Present

[55] Well-developed pair of genal spines on cephalic shield

0 . Absent

1. Present

Remark: We code this character as present in Sklerolibyon, although the spines extend from the fused plate of the first two segments of the shield, and not from

[56] Cephalic shield overlapping first trunk sternite, pair of trunk appendages, or trunk caeca

0 . Absent

1. Overlap of sixth body somite

2. Overlap of eighth body somite

Remark: See Mayers et al. (2018, ch. 22). This is a multistate character without corresponding sovereign character because states 1 and 2 are unlikely to have a common origin.

[57] Dorsal facial sutures

0 . Absent

1. Present

BRAIN CHARACTERS

[58] Ganglia of post-oral cephalic appendages fused into single nerve mass [AC47]

0 . Absent

1. Present

[59] Trunk ganglia individually expressed*

0 . Absent

1. Present

Remark: Generalized from Wang et al. (2018), ch. 146.

[60] Contiguity of the first two post-protocerebral ganglia [AC48]

0 . Absent

1. Present

[61] Fan-shaped body in brain [AC49]

0 . Absent

1. Present

[62] Position of midline neuropil [AC50]

0 . Superficial to protocerebrum

1. Embedded within protocerebral matrix

[63] Olfactory lobes linked to a lateral component of protocerebrum by olfactory globular tract [AC51] 
0. Absent

1. Present

[64] Deutocerebral olfactory lobe with glomeruli [AC52]

0 . Absent

1. Present

[65] Lateral eyes pedunculated [AC53]

0 . Absent

1. Present

\section{STERNITES (CEPHALON)}

[66] Sternites [AC54]

0 . Absent

1. Present

[67] Endosternum (or endosternite) [AC55]

0 . Absent

1. Present

[68] Labrum*

0. Absent

1. Present

Remark: Characters describing the hypostome-labrum complex have been reorganized and expanded compared to Aria and Caron (2017a). Here, we code the labrum as a common feature shared by all euarthropods, which arguably originates from the protocerebral somite (Scholtz and Edgecombe 2006). Classically, in mandibulates, the "labrum" covers the mouth and such pre-oral structure generally takes two forms: the typical fleshy protrusion of oligostracans, anostracans and other crustaceans, and the sclerotic plates encountered in myriapods, malacostracans and hexapods-which are commonly designated as either "epistome-labrum" or "clypeo-labrum" depending on the group of interest. Pre-labral sclerites in mandibulates are often distinct from the labrum per se and are usually closely associated with the insertion of the antennules. For this reason, the pre-labral sclerite is sometimes referred to as the "hypostome," a common term from the pre-oral sclerite used among extinct euarthropods, especially trilobites and their allies. Such a terminology has been used for instance for Cephalocarida (Olesen et al. 2011) but arguably applies to all other crustaceans, including copepods (Schram 1986), leptostracans (Olesen and Walossek 2000) and stomatopods (Haug et al. 2012a). Herein (chars. 62-64), we therefore harmonize the terms "epistome," "clypeus" and "hypostome" under the same concept of "hypostome."

[69] Labrum expression and location*

0 . Expressed frontally

1. Expressed as a postero-ventral structure separate from frontalmost organs 
Remark: This character fundamentally distinguishes the anteriormost chelicerate "epistomelabrum" from ventral structures characteristic of Mandibulata. Hymenocarines and ostracods are considered to belong to the first group (see Vannier et al., 2018).

[70] External inter-ocular sensory organs [AC68 $]^{*}$

0 . Absent

1. Present

Remark: Ortega-Hernández and Budd (2016) have discussed their views on the analogous nature of sensory organs and paired inter-ocular projections in panarthropods. Our aim here is to test local cases of homology, and we therefore code this character as present when known. We do however make a distinction for the limb-like inter-ocular features of Canadaspis and remipedes (see char. 86).

[71] Type of ventral labrum* [AC58]

0 . Single fleshy protrusion

1. Plate with underlying soft tissues

[72] Labral plate, type*

0 . Bilobate lip with partial fusion to the hypostome

1. Free bilobate or wide lip

2. Expanded subtriangular or subrectangular lip

Remark: This character codes for variations in labral shape amongst mandibulates. Most crustaceans possess state 2 , whereas myriapods possess state 0 . State 1 is derived and found in pterygotes.

[73] Hypostome [AC56]*

0 . Absent

1. Present

Remark: The hypostome is here defined on the basis of the artiopodan hypostome, that is, as a preoral sclerotic structure associated with the insertion of the anteriormost appendages.

[74] Hypostome attachment [AC57]*

0 . Conterminant

1. Natant

[75] Hypostome accommodating antennules and extensively covering the mouth [AC59]

0 . Absent

1. Present

Remark: This is a character mostly discriminating the typical artiopodan hypostome.

[76] Mandibulate labium [AC60]

0. Absent 


\section{Present}

[77] Chelicerate labium*

0. Absent

1. Present

Remark: The chelicerate labium is a ventral sclerite (sternapophysis) apparently associated with the palpal somite and forming the posterior border of the stomodaeum. We follow Wang et al. (2018) in coding the labium as absent in Pedipalpi but otherwise code state 1 for pseudoscorpions and solifuges after Schultz (2007).

[78] Sternites externally developed within segments 2-4 [AC61]

0 . Absent

1. Present

[79] Fusion of sternites within segments 2-4 [AC62]

0 . Absent

1. Present

[80] Metastoma [AC63]*

0. Absent

1. Present

Remark: The metastoma is a modified sternite of the first opisthosomal segment in euchelicerates - typically, in eurypterids (Dunlop 1997). We also code this character for scorpions (sternum) and harvestmen (aculi genitales) (Dunlop and Lamsdell 2016).

[81] Coxosternite [AC64]

0 . Absent

1. Present

[82] Single main maxilliped [AC163]

0 . Absent

1. Present

[83] Head has tendention to form a hypostomal bridge [AC65]

0 . Absent

1. Present

[84] First opisthosomal tergite triangular

0 . Absent

1. Present

Remark: See Wang et al. (2018), ch. 109. Only applies to the total-group Chelicerata given that the homology of this somite requires a prosoma. 
[85] Genital operculum overlaps sternite of third opisthosomal segment

0 . Absent

1. Present

Remark: See Wang et al. (2018), ch. 123. Only applies to the total-group Chelicerata given that the homology of this somite requires a prosoma.

FRONTALMOST APPENDAGES

[86] Frontalmost limb-like projections

0 . Absent

1. Present

Remark: This character codes specifically for the appendicular outgrowths present between the eyes of Canadaspis, and comparable ones in remipedes. Their nature and homology with other known cephalic features remain uncertain.

[87] Orientation of first axial appendage [AC73]

0 . Ventro-frontal

1. Dorsal

[88] Arthrodization of first axial appendage [AC66]

0 . Absent

1. Present

[89] Size of frontalmost appendage [AC74]

0 . Long, multipodomerous; $>6$ podomeres

1. Short $\leq 6$ podomeres

[90] Well-developed ventral spinose outgrowths on frontalmost appendage [AC76]

0 . Absent

1. Present

[91] Rows of inner (ventral) spines on frontalmost appendage

0 . Single row

1. Double row ("Anomalocaris type")

[92] Type of inner (ventral) spinose outgrowths on frontalmost appendage (dependent on char. 94) [AC77]

0 . Sub-equal length or tapering gradually along entire margin

1. Elongate mid-margin

[93] Secondary spines on ventral spinose outgrowths of frontalmost appendage [AC78]

0 . Absent

1. Present

[94] Outgrowths forming elongate rami on frontalmost appendage [AC69] 
0. Absent

1. Present

[95] Rami of branching frontalmost appendage originating from different podomeres [AC70]

0 . Absent

1. Present

[96] Short and ramified frontalmost appendage ending in a raptorial device made of three to four elongate spines*

0 . Absent

1. Present

Remark: This is a potential apomorphy of Megacheira.

[97] Spine morphology of megacheiran appendage*

0 . Thin, ca. 10 times as long as thin (base width) or more

1. Thick, ca. 7 times as long as thin (base width) or less

[98] Multichelate device type on megacheiran appendage*

0 . Made of four spines ('yohoiid type')

1. Made of three spines ('leanchoiliid type')

[99] Podomere number of articulating basis of megacheiran appendage*

0 . Single elongate podomere

1. Two short podomeres

[100] Single-podomere basis of megacheiran appendage, type*

0 . Slender

1. Stout

Remark: Fortiforceps and apparently also Parapeytoia possess a stouter basal podomere compared to other megacheirans with yohoiid type of "great appendages."

[101] Shape of peduncular podomere for yohoiid great appendages*

0 . Sub-cylindrical

1. Chalice-shaped

[102] Thickness of peduncular podomere for yohoiid great appendages*

0 . Slender

1. Stout

Remark: Yohoia and Jianfengia have slenderer peduncles compared to other megacheirans. A Yohoia morph was reported (Haug et al. 2012b) to have stouter 'great appendages,' but appears less common-it may also be a different species than Yohoia tenuis.

[103] Ramified frontalmost appendage with flagellate extensions [AC71] 
0. Absent

1. Present

[104] Short frontalmost appendage with first and second podomere forming an elbowed articulation*

0 . Absent

1. Present

Remark: Typical of non-leanchoiliid megacheirans and some chelicerates, see Haug et al. (2012b) and Wang et al. (2018), ch. 37.

[105] Frontalmost appendage a chelicera, i.e. chelate or subchelate with only two opposing faces [AC72]

0 . Absent

1. Present

[106] Orientation of closure of terminal podomere (apotele) on chelicera*

0 . Ventral

1. Dorsal

2. Lateral

Remark: See Wang et al. (2018), ch. 40.

[107] Cheliceral fang*

0 . Absent

1. Present

Remark: See Wang et al. (2018), ch. 41.

[108] Number of cheliceral podomeres*

0 . Three

1. Two

Remark: See Wang et al. (2018), ch. 35.

[109] Cheliceral serrula*

0 . Absent

1. Present

Remark: See Wang et al. (2018), ch. 53.

[110] Arthrodized frontalmost appendage, multipodomerous type [AC75]

0. Robust, thick branch

1. Long antennular

[111] Dorsal spinose outgrowths on podomeres of arthrodized frontalmost appendage [AC79] 
0. Absent

1. Present

OTHER CEPHALIC LIMBS

[112] Maximum podomere number in head (tagma I) [AC90]*

0.7

1. $<7$

2. $>7$

Remark: When endopods are differentiated and vary from one somite to the other, this character takes into account the endopod with the highest number of podomeres.

[113] Punctual subdivision of endopod podomeres from an heptopodomeran limb*

0. Absent

1. Basi- and telofemur

2. Basi- and telotarsus

Remark: See Wang et al. (2018), chs. 88 and 93. This multistate has no sovereign character because states 1 and 2 do not necessarily have a common origin.

[114] All cephalic endopods posterior to frontalmost appendage pair well-developed (sevensegmented or more) [AC81]*

0 . Absent

1. Present

Remark: Taking the heptopodomeran condition as a reference for the ground pattern (see Aria et al. 2015), endopods are considered reduced here when the podomere count is below seven. Sometimes an exact podomere count has not been possible so far (e.g. in the first post-antennular limb of leanchoiliids) and the modified state is occasionally extrapolated from a substantial reduction of the appendage in size.

[115] Endopod of second appendage pair [AC82]

0 . Developed

1. Reduced

[116] Endopod of third appendage pair [AC83]

0. Developed

1. Reduced

[117] Endopod of fourth appendage pair [AC84]

0. Developed

1. Reduced

[118] Exopod of fourth appendage pair [AC112]*

0. Developed

1. Reduced 
[119] Endopod of fifth appendage pair [AC85]

0. Developed

1. Reduced

[120] Exopods of fifth appendage pair*

0 . Developed

1. Reduced

[121] Exopods on cephalic appendages posterior to fifth pair*

0 . Absent

1. Present

[122] Exopod of cephalic appendages excluding two anteriormost pairs, type [AC102]

0 . Stenopodous, podomeres $>4$

1. Annulate

2. Short, few podomeres $(\leq 4)$

Remark: See Mayers et al. (2018).

[123] Subdivision of short cephalic exopod, type

0 . Bipartite

1. Tripartite

Remark: See Mayers et al. (2018).

[124] Stenopodous exopod, type [AC103]

0 . Antenniform with elongate podomeres

1. Short and stout, ending in setal brush (underdeveloped claw)

[125] Partial detachment of exopods from main limb branch in head tagma*

0 . Absent

1. Present

Remark: This character expresses the peculiar condition of habeliidans, Offacolus and Dibasterium, in which the cephalic exopods preserve as partially dissociated from their main biramous branch (Aria and Caron 2017b). The exact attachment remains unknown.

[126] Some cephalic endopods are walking limbs [AC86]

0 . Absent

1. Present

[127] Repeated appendage morphology in tagma I [AC87]

0 . Absent

1. Present 
[128] Dichotomy in appendage morphology between tagma I and tagma II [AC88]

0 . Absent

1. Present

[129] Proximo-distal differentiation of endopod podomeres in head (tagma I) [AC89]

0 . Absent

1. Present

[130] Arthrodized post-antennular appendage expressed [AC91]

0 . Absent

1. Present

[131] Post-antennular appendage differentiated [AC92]

0 . Absent

1. Present

[132] Chelate or sub-chelate termination of post-antennular appendage [AC93]*

0 . Absent

1. Present

[133] Plane of motion of chelate or sub-chelate post-antennular appendage*

0 . Sub-vertical, leg-like

1. Horizontal

Remark: See Wang et al. (2018), ch. 62.

[134] Ramification of post-antennular appendage [AC95]

0 . Uniramous

1. Biramous

[135] Developed endites on endopod of post-antennular appendage [AC96]

0 . Absent

1. Present

[136] Endopod of post-antennular appendage annulate or flagellate [AC97]

0 . Absent

1. Present

[137] Coxa on post-antennular appendage [AC99]

0 . Absent

1. Present

[138] Exopod of post-antennular appendage, type [AC100]

0 . Stenopodous

1. Annulate

2. Short, lobate 
[139] Endopod of third cephalic appendage very thin and elongate, filament-like*

0. Absent

1. Present

Remark: This condition is sometimes called "antenniform" in chelicerates (e.g. Dunlop and Lamsdell 2016). We differentiate the chelicerate condition from the annulate condition, that is, with podomeres extremely thin and elongate, as opposed to being very short and numerous.

[140] Enditic outgrowths on cephalic endopods excluding two anteriormost pairs [AC104]

0 . Absent

1. Present

[141] Endopod of third cephalic appendage chelate or subchelate [AC105]

0 . Absent

1. Present

[142] Third cephalic appendage with a well-developed, toothed gnathobase [AC106]

0 . Absent

1. Present

[143] Third cephalic appendage a mandible [AC107]

0 . Absent

1. Present

[144] Mandibular palp [AC107]

0 . Non-developed

1. Developed

[145] Mandible with three-segmented palp, appressed on the ventral side of the head, curving inward [AC94]*

0 . Absent

1. Present

Remark: This characterizes the endopod of the fuxianhuiid mandible.

[146] Telognathic mandible [AC108]

0 . Absent

1. Present

[147] Mandibular gnathal edge [AC109]

0 . Consisting of molar and incisor process

1. Only ellipsoid pars molaris present

2. Row of parallel teeth

3. Shovel with terminal teeth

4. Group of paired teeth and hair pad 
[148] Mandibular lamellate combs [AC110]

0 . Absent

1. Present

[149] Hypopharynx [AC111]

0 . Absent

1. Present

[150] Modified endopod/palp on fourth cephalic appendage [AC113]

0 . Absent

1. Present

Remark: This character implies the modification of the appendage basis as a mouthpart and the reduction of the endopod of the fourth appendage pair (char. 90), whereby the complete reduction of the endopod is coded " 0 ".

[151] Modified endopod/palp on fourth cephalic appendage, type [AC114]*

0 . Reduced, vestigial, undeveloped

1. Well developed

[152] Post-mandibular plate formed by the fusion of the maxilla and the intermaxillary sternum [AC115]

0 . Absent

1. Present

[153] Cephalic appendages 4 and 5 ending with chelate termination [AC116]

0 . Absent

1. Present

[154] Fifth cephalic appendage, differentiation type [AC117]

0 . Integrated to gnathal plate (labium)

1. Reduced, enditic

[155] Fifth cephalic appendage vestigial [AC118]

0 . Absent

1. Present

[156] Fifth cephalic appendage with developed palp [AC119]

0 . Absent

1. Present

Remark: Same requirements for coding as for char. 121, but with respect to fifth cephalic pair.

[157] Internalization of mouthparts [AC120]

0 . Absent 


\section{Present}

[158] Oral cone [AC121]

0 . Absent

1. Present

[159] Atrium oris [AC122]

0 . Absent

1. Present

MOUTH AND STOMODAEAL AREA

[160] Mouth opening [AC123]

0 . Frontal

1. Ventral

[161] Ventral mouth opening, type

0 . Antero-ventral

1. Postero-ventral

[162] Type of circumoral structures [AC124]*

0 . Toothed lips

1. Lamellae

2. Ring of plates

3. Mouth surrounded by arthrodized limbs

[163] Circumoral structures sclerotized [AC125]*

0 . Absent

1. Present

[164] Proboscis [AC126]

0 . Absent

1. Present

ALIMENTARY TRACT AND OTHER INTERNAL CHARACTERS

[165] Large and well-differentiated stomach [AC129]

0 . Absent

1. Present

[166] Stomach in a frontal position [AC131]

0 . Absent

1. Present

[167] Stomach —additional pouch (crop) [AC132]

0 . Absent

1. Present 
[168] Secondary organs connected to the central digestive duct [AC132]

0 . Absent

1. Present

[169] Secondary digestive organs serially repeated along the post-cephalic portion of the gut [AC133]

0 . Absent

1. Present

[170] Shape of post-cephalic secondary digestive structures [AC134]

0 . Reniform

1. Bulgy triangles

2. Caeca

[171] Striations on post-cephalic secondary digestive structures [AC135]

0. Absent

1. Present

[172] Branching of post-cephalic secondary digestive structures [AC136]

0 . Absent

1. Present

[173] Differentiation of cephalic secondary digestive structures (compared to trunk) [AC137]

0 . Absent

1. Present

[174] Cephalic secondary digestive structures sharing a single base connecting to the main intestinal tract [AC138]
0. Absent
1. Present

[175] Branching of cephalic secondary digestive structures [AC139]

0 . Absent

1. Present

[176] Peritrophic membrane [AC140]

0 . Absent

1. Present

[177] Metameric ganglia on nerve cord [AC141]

0 . Absent

1. Present

[178] Metanephridia with sacculus containing podocytes [AC142]

0 . Absent 
1. Present

[179] Segmental invaginations of neuroectoderm giving rise to ventral organs [AC143]

0 . Absent

1. Present

[180] Malpighian tubules*

0 . Absent

1. Present

Remark: See Wang et al. (2018), ch. 164, for coding in arachnids.

[181] Coxal glands opening at base of prosomal leg $1^{*}$

0 . Absent

1. Present

Remark: See Wang et al. (2018), ch. 166. Only applies to Euchelicerata.

[182] Distodorsal insertion of posterior transpatellar muscle*

0. Absent

1. Present

Remark: See Wang et al. (2018), ch. 190. Muscle data in chelicerates carry some obvious conflicts, both internally and with other parts of the anatomy. We selected those characters based on musculature that provide information on major internal arachnid subdivisions (aside from the extensive evidence supporting the monophyly of Arachnida and Pedipalpi, methodologically enforced in our analysis) but do not assume spurious configurations, such as a polyphyletic tetrapulmonata. We also kept the information to the homologous insertions of the muscles and did not assume losses as having common origins in case of multistates. Only applies to Euchelicerata.

[183] Ventral insertion of the anterior transpatellar muscle*

0 . Absent

1. Present

Remark: See Wang et al. (2018), ch. 192. Only applies to Euchelicerata.

[184] Ventral insertion of the anterior patellotibial muscle on tibia*

0 . Absent

1. Present

Remark: See Wang et al. (2018), ch. 193. Only applies to Euchelicerata.

[185] Posterior patellotibial muscle*

0 . Absent

1. Present 
Remark: See Wang et al. (2018), ch. 194. Only applies to Euchelicerata.

[186] 9x2 +3 microtubule arrangement in euchelicerate sperm axoneme*

0 . Absent

1. Present

Remark: See Wang et al. (2018), ch. 218. Only applies to Euchelicerata.

[187] Iso/telolecithal euchelicerate eggs*

0 . Absent

1. Present

Remark: See Wang et al. (2018), ch. 226. Only applies to Euchelicerata.

TRUNK

[188] Fusion of posterior trunk tergites

0 . Absent

1. Present

Remark: See Mayers et al. (2018, ch. 2).

[189] Fusion of posterior trunk tergites, type

0 . Partial—-some segments still freely articulate

1. Complete - all trunk tergites show some degree of fusion

Remark: See Mayers et al. (2018, ch. 3).

[190] Degree of effacement of tergite boundaries on fused trunk portion

0 . Low-pleurae outstanding, at least laterally

1. High - pleurae margins fused, at least laterally

2. Tergo-pleurae individualized and arthrodial membranes sometimes visible, but with no tergite overlap

Remark: See Mayers et al. (2018, ch. 5). State 2 is added here to reflect the condition of most terrestrial euarthropods. Certain chelicerates (Haptopoda, Uropygi) have fused trunks but retain overlapping tergites.

[191] Distinct pygidium [AC209]

0 . Absent

1. Present

Remark: See Mayers et al. (2018, ch. 7).

[192] Thorax [AC144]

0 . Absent

1. Present 
[193] Number of thoracic somites [AC145]

0 . $11+$

1. $4 / 5$

2. $6 / 9$

3. 3

[194] Abdomen [AC146]

0 . Absent

1. Present

[195] Post-abdomen

0 . Absent

1. Present

Remark: A post-abdomen is defined here by the differentiation of limbless tergo-pleurae posterior to an abdomen.

[196] Multisegmentation (trunk somites $\geq 20$ ) [AC149]

0. Absent

1. Present

[197] Number of core trunk segments (non-multisegmented taxa) [AC147]*

0. $15-19$

1. $10-14$

2. 9

3. $7-8$

4. $<7$

[198] Seventh appendage integrated into the prosoma [AC148]*

0 . Absent

1. Present

Remark: This character only applies to Chelicerata and their stem groups (see also char. 32).

[199] Tergite of eighth somite (counting the ocular somite as the first) drastically reduced as a "microtergite"*

0 . Absent

1. Present

Remark: See Dunlop and Lamsdell (2016) for a review of this character across chelicerates.

[200] Constriction of eighth somite (segment seven) into a pedicel*

0 . Absent

1. Present 
Remark: A widespread arachnid character (see e.g. Dunlop and Lamsdell (2016)).

[201] Post-cephalic appendages covered by sclerotic plates (opercula)*

0 . Absent

1. Present

Remark: Used as an apomorphy of Euchelicerata (Aria and Caron 2017b, Dunlop and Lamsdell 2016), although it mostly applies to merostomes and tetrapulmonates.

[202] Tergo-sternal decoupling [AC150]

0 . Absent

1. Present

[203] Tergo-sternal decoupling, type [AC151]

0 . Polypody

1. Polypody and "polysternity"

2. "Polytergity" (autapomorphy of symphylan myriapods)

[204] Pleurae [AC152]

0 . Reduced or fused

1. Developed

[205] Tergo-pleural rings [AC153]*

0 . Absent

1. Present

[206] Pleural orientation [AC154]

0 . Horizontal

1. Around body

[207] Pleural length [AC155]

0 . Short, i.e. equal or inferior to body diameter

1. Long, i.e. exceeding body diameter

[208] Articulating ridge [AC156]

0 . Absent

1. Present

[209] Articulating ridge, type [AC157]

0 . Single

1. Antero-posterior

[210] Transverse stipital muscle [AC158]

0 . Absent

1. Present

TRUNK APPENDAGES AND GENERAL APPENDICULAR CHARACTERS 
[211] Limb arthrodization in trunk [AC181]

0 . Absent

1. Present

[212] Proximo-distal differentiation of endopod podomeres in tagma II [AC159]

0 . Absent

1. Present

[213] Podomere number in endopods of tagma II [AC160]

0.7

1. $<7$

2. $>7$

[214] Maxillipeds [AC161]

0 . Absent

1. Present

[215] Tergites of maxilliped segments fused to head shield [AC162]

0 . Absent

1. Present

[216] Maxilliped pairs*

0 . Single main pair

1. Several pairs

[217] Slit sensilla [AC164]

0 . Absent

1. Present

[218] Trichobothria*

0 . Absent

1. Present

Remark: See Wang et al. (2018), ch. 139.

[219] Basis (basipod) [AC165]

0 . Absent

1. Present

[220] Basipod formed of at least two elements [AC166]

0 . Absent

1. Present

[221] Basipod multi-segmented [AC167]

0 . Absent 


\section{Present}

[222] Multiple endites on basipod [AC168]

0 . Absent

1. Present

[223] Proximalmost endite on basipod [AC169]

0 . Absent

1. Present

[224] Coxa as entire pre-basal podomere [AC170]

0 . Absent

1. Present

[225] Precoxa as whole pre-coxal podomere [AC171]

0 . Absent

1. Present

[226] Pleurites formed by several sclerotic elements surrounding limb insertion [AC172]

0 . Absent

1. Present

[227] Arrangement of pleurites [AC173]

0 . Outer/proximal and distal/inner sets

1. Multiple sclerotic pieces

[228] Gnathobases (= heavily-sclerotized and toothed masticatory basipods) on any body limb [AC174]

0 . Absent

1. Present

[229] One or more gnathobase(s) reduced in tagma I [AC175]

0 . Absent

1. Present

[230] Secondary appendicular outgrowths on trunk [AC176]

0 . Absent

1. Present

[231] Secondary appendicular outgrowths on trunk, type [AC177]

0. Lobopodous

1. Sclerotized

[232] Distal oblanceolate lamellae on exopod

0 . Absent

1. Present 
[233] Proximal lamellae (gills) with appendicular affinity in trunk [AC178]

0 . Absent

1. Present

[234] Proximal lamellae (gills) present on trunk somites 10 to $13^{*}$

0 . Absent

1. Present

Remark: Generalized from Wang et al. (2018), ch. 152, to all relevant arthropods.

[235] Book gills made of a series of broad, overlapping lamellae*

0 . Absent

1. Present

Remark: This character codes for the gill form generally characteristic of euchelicerates, worded to also include variations with fewer lamellae than those known in merostomes.

[236] Proximal lamellae internalized [AC179]

0 . Absent

1. Present

[237] Trunk exopod posterior to head tagma, type [AC182]*

0 . Reduced, vestigial

1. Annulate

2. Short, few podomeres $(\leq 4)$

3. Phyllopodous

[238] Short trunk exopod, type

0 . Single lobe

1. Bipartite

2. Tripartite

[239] Trunk endopod reduced posterior to head tagma [AC180]

0 . Absent

1. Present

[240] Endopod strongly developed in thorax (or anterior trunk if thorax undifferentiated) [AC183]

0 . Absent

1. Present

[241] Phyllopodous-type limbs anywhere on body [AC184]

0 . Absent

1. Present 
[242] Terminal endopods stenopodous [AC185]

0 . Absent

1. Present

[243] Identical morphology of endopod and exopod rami on pleopods/post-thorax [AC186]

0 . Absent

1. Present

[244] Annulation of at least one pair of exopods [AC187]

0. Absent

1. Present

[245] Epipod [AC191]

0. Absent

1. Present

[246] Endites as latero-distal projections on endopod podomeres [AC192]

0 . Absent

1. Present

[247] Pusher legs with paddle tips [AC193]

0 . Absent

1. Present

[248] Developed endites on endopod podomeres in trunk (tagma II and III) [AC194]

0. Absent

1. Present

[249] Paired spines on endopod podomeres [AC195]

0 . Absent

1. Present

[250] Short spines on endopod podomeres [AC196]

0 . Absent

1. Present

[251] Multiple setae on endopod podomeres [AC197]

0 . Absent

1. Present

[252] Complex articulation between basipod and first endopod podomere involving surnumerary sclerites*

0. Absent

1. Present 
Remark: Generalized from Wang et al. (2018), ch. 87. Mandibulate pleurites are not considered here.

[253] Bicondylar femoropatellal articulation*

0 . Absent

1. Present

Remark: See Wang et al. (2018), ch. 89. Only applicable to euchelicerates.

[254] Patellotibial articulation*

0 . Monocondylar

1. Hinge

2. Bicondylar

Remark: See Wang et al. (2018), ch. 90. Only applicable to euchelicerates.

[255] Main limb tip along body [AC199]

0 . Pad

1. Juxtaposed claws

2. Trident of claws

3. Chelate or sub-chelate

4. Double claw

5. Multiple spines

6. Single claw

[256] Coxal vesicles*

0 . Absent

1. Present

Remark: So-called "sternal pores" in euthycarcinoids have been reinterpreted by Edgecombe and Morgan (1999) as probable homologs to the coxal vesicles of certain terrestrial mandibulates, including Symphyla and Diplopoda. We follow this view here.

POSTERIOR TERMINATION

[257] Sclerotization of termination [AC200]

0 . Absent

1. Present

[258] Telson developed [AC201]

0 . Absent

1. Present

[259] Telson type [AC202]

0 . Spine

1. Plate / Spatula 
[260] Flagellate extension of telson*

0 . Absent

1. Present

[261] Anus location [AC203]

0 . Terminal somite

1. Base of telson

[262] Caudal rami [AC204]

0 . Absent

1. Present

[263] Caudal rami, type [AC208]

0 . Spinose

1. Annulate

2. Subdivided

3. Rounded

4. Flap

[264] Additional caudal processes [AC205]

0 . Absent

1. Present

[265] Furca [AC206]

0 . Absent

1. Present

[266] Uropods sensu stricto [AC207]

0 . Absent

1. Present

[267] Axial elevation of pygidium [AC211]

0 . Absent

1. Present

[268] Pygidial ornamentation [AC212]

0 . Smooth

1. Spinose

\section{Supplementary references}

Aria, C., and J.-B. Caron. 2015. Cephalic and limb anatomy of a new isoxyid from the Burgess Shale and the role of "stem bivalved arthropods" in the disparity of the frontalmost appendage. PLOS ONE 10(6): e0124979. 
Aria, C., and J.-B. Caron. 2017a. Burgess Shale fossils illustrate the origin of the mandibulate body plan. Nature 545:89-92.

Aria, C., and J.-B. Caron. 2017b. Mandibulate convergence in an armoured Cambrian stem chelicerate. BMC Evolutionary Biology 17:261.

Aria, C., and J. B. Caron. 2019. A middle Cambrian arthropod with chelicerae and proto-book gills. Nature 573:586-589.

Briggs, D. E. G., R. A. Fortey, and M. A. Wills. 1992. Morphological disparity in the Cambrian. SCIENCE 256(5064):1670-1673.

Caron, J.-B., and C. Aria. 2017. Cambrian suspension-feeding lobopodians and the early radiation of panarthropods. BMC Evolutionary Biology 17:29.

Dunlop, J. A. 1997. The origins of tetrapulmonate book lungs and their significance for chelicerate phylogeny. In P. A. Selden, ed. Proceedings of the 17th European Colloquium of Arachnology. Edinburgh.

Dunlop, J. A., and J. C. Lamsdell. 2016. Segmentation and tagmosis in Chelicerata. Arthropod Structure \& Development 46(3):395-418.

Edgecombe, G. D., and H. Morgan. 1999. Synaustrus and the euthycarcinoid puzzle. Alcheringa 23(3-4):193-213.

Haug, C., W. S. Sallam, A. Maas, D. Waloszek, V. Kutschera, and J. T. Haug. 2012a. Tagmatization in Stomatopoda - reconsidering functional units of modern-day mantis shrimps (Verunipeltata, Hoplocarida) and implications for the interpretation of fossils. Frontiers in Zoology 9:31.

Haug, J. T., D. Waloszek, A. Maas, Y. Liu, and C. Haug. 2012b. Functional morphology, ontogeny and evolution of mantis shrimp-like predators in the Cambrian. Palaeontology 55:369-399.

Lee, M. S. Y., J. Soubrier, and G. D. Edgecombe. 2013. Rates of phenotypic and genomic evolution during the Cambrian Explosion. Current Biology 23(19):1889-95.

Legg, D. A., M. D. Sutton, and G. D. Edgecombe. 2013. Arthropod fossil data increase congruence of morphological and molecular phylogenies. Nature Communications $4: 2485$.

Lloyd, G. T. 2016. Estimating morphological diversity and tempo with discrete character-taxon matrices: implementation, challenges, progress, and future directions. Biological Journal of the Linnean Society 118(1):131-151.

Olesen, J., J. T. Haug, A. Maas, and D. Waloszek. 2011. External morphology of Lightiella monniotae (Crustacea, Cephalocarida) in the light of Cambrian 'Orsten' crustaceans. Arthropod Structure \& Development 40(5):449-478.

Olesen, J., and D. Walossek. 2000. Limb ontogeny and trunk segmentation in Nebalia species (Crustacea, Malacostraca, Leptostraca). Zoomorphology 120(1):47-64.

Ortega-Hernandez, J., and G. E. Budd. 2016. The nature of non-appendicular anterior paired projections in Palaeozoic total-group Euarthropoda. Arthropod Structure \& Development 45(2):185-199.

Rota-Stabelli, O., L. Campbell, H. Brinkmann, G. D. Edgecombe, S. J. Longhorn, K. J. Peterson, D. Pisani, H. Philippe, and M. J. Telford. 2011. A congruent solution to arthropod phylogeny: phylogenomics, microRNAs and morphology support monophyletic Mandibulata. Proceedings of the Royal Society B-Biological Sciences 278(1703):298306. 
Scholtz, G., and G. D. Edgecombe. 2006. The evolution of arthropod heads: reconciling morphological, developmental and palaeontological evidence. Development Genes And Evolution 216(7-8):395-415.

Schram, F. R. 1986. Crustacea. Oxford University Press.

Shultz, J. W. 2007. A phylogenetic analysis of the arachnid orders based on morphological characters. Zoological Journal of the Linnean Society 150(2):221-265.

Vannier, J., C. Aria, R. S. Taylor, and J.-B. Caron. 2018. Waptia fieldensis Walcott, a mandibulate arthropod from the middle Cambrian Burgess Shale. Royal Society Open Science 5:172206.

Wang, B., J. A. Dunlop, P. A. Selden, R. J. Garwood, W. A. Shear, P. Muller, and X. Lei. 2018. Cretaceous arachnid Chimerarachne yingi gen. et sp. nov. illuminates spider origins. Nature Ecology and Evolution 2(4):614-622. 